\title{
Mechanical Stability of Lipid Membranes Decorated with Dextran Sulfate
}

\author{
Candelaria I. Cámara, ${ }^{\dagger, \ddagger}$ Florencia E. Lurgo, ${ }^{\dagger \neq}$ Maria Laura Fanani, ${ }^{\dagger, \ddagger(0)}$ and Natalia Wilke ${ }^{*, \dagger, \ddagger(0)}$ \\ ${ }^{\dagger}$ Facultad de Ciencias Químicas, Departamento de Química Biológica Ranwel Caputto, Ciudad Universitaria, Universidad Nacional \\ de Córdoba, X5000HUA Córdoba, Argentina \\ *Centro de Investigaciones en Química Biológica de Córdoba (CIQUIBIC), Ciudad Universitaria, CONICET, Universidad \\ Nacional de Córdoba, X5000HUA Córdoba, Argentina
}

\section{Supporting Information}

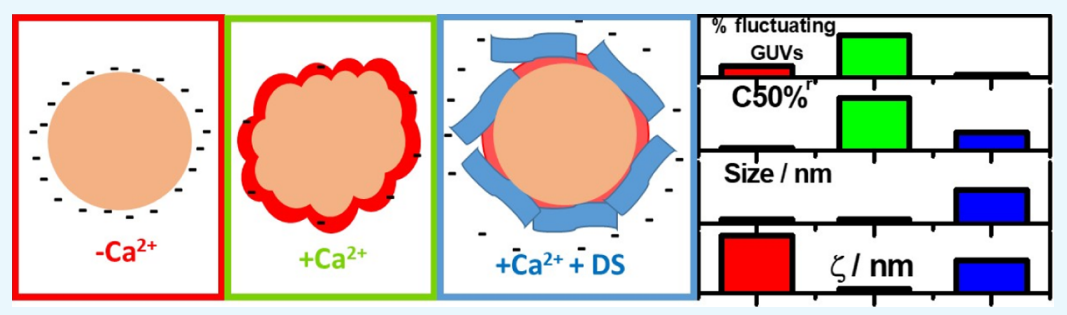

ABSTRACT: Lipid vesicles decorated with polysaccharides have been proposed as vehicles for drug delivery because the polymers confer to the vesicles an enhanced stability, increasing the probability of the drug for reaching the target cell. Here, we first test the affinity of dextran sulfate (DS) for two different vesicle composition, and afterward, we study the effect of DS on the liposome mechanical properties. We found that DS binds to both tested membrane compositions. The interaction of DS with the anionic membranes studied here is mediated by the metal ions present in the aqueous solution $\left(\mathrm{Na}^{+}\right.$and $\left.\mathrm{Ca}^{2+}\right)$, being higher in the presence of $\mathrm{Ca}^{2+}$. Binding occurs preferentially in regions of closely packed lipids. Strikingly, DS did not affect the stability against detergent and the membrane rigidity of none of the vesicles. Thus, the proposed stability increase induced by this kind of polymers in drug delivery systems is not related with a modulation of the membrane thermodynamic properties but to other biochemical factors.

\section{INTRODUCTION}

Liposomes have been largely proposed as vehicles for controlled drug delivery. The work of several researchers over almost 5 decades led to the development of important technical advances, leading to numerous clinical trials in such diverse areas as the delivery of anticancer, antifungal, and antibiotic drugs, gene medicines, anesthetics, and antiinflammatory drugs.

In order to efficiently deliver the drug, liposomes have to be stable upon permeation and degradation until the target is reached. In this regard, polysaccharide-coated liposomes have been appreciated of being physically stable systems against biochemical and physicochemical stresses encountered in biofluids especially after oral administration. This kind of coated-vesicles showed reduced permeability to water-soluble encapsulated materials in the presence of blood plasma or serum, increased stability against enzymatic attack, and mechanical stability toward biostimuli. ${ }^{2}$

Among the tested polysaccharides, dextran-derivative polymers have been widely studied, and it was found that conjugation of liposomes with them results in favorable alteration of pharmacokinetics and pharmacodynamics of therapeutic agents. ${ }^{1,3-8}$ In this context, it is important to know at which level the polymer acts on the liposome properties.
Dextran comprises a family of an $\alpha-(1 \rightarrow 6)$ linked Dglucose main chain with varying proportions of linkages and branches. They are versatile because of their solubility, biocompatibility, and biodegradability. ${ }^{9}$ Their hydroxyl groups offer many sites for derivatization, giving rise to a large number of polysaccharides, among which is the polymer used in this study, dextran sulfate (DS). This polymer is a polyelectrolyte with sulfate groups along its chain (see Figure 1), which provides a wide range of applications such as an anticoagulant, an inhibitor of human immunodeficiency virus and herpes simplex virus, and a reducer of cancer metastasis and tumor adherence. ${ }^{9,10}$

The structure of DS resembles that of the glycosaminoglycans present in the extracellular matrix, thus the coating of these polymers on liposomes may help hiding the vesicle from the immune system and protect liposomes from biodegradation, thereby increasing the permanence of intact vesicles in the bloodstream. ${ }^{11}$ On the other hand, polymers may stabilize the liposomes thermodynamically, increasing the mechanical stability of the membranes.

Received: July 4, 2018

Accepted: September 10, 2018

Published: September 24, 2018 
(a)

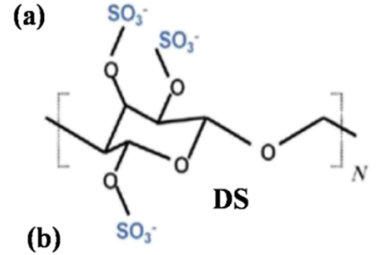

(b)
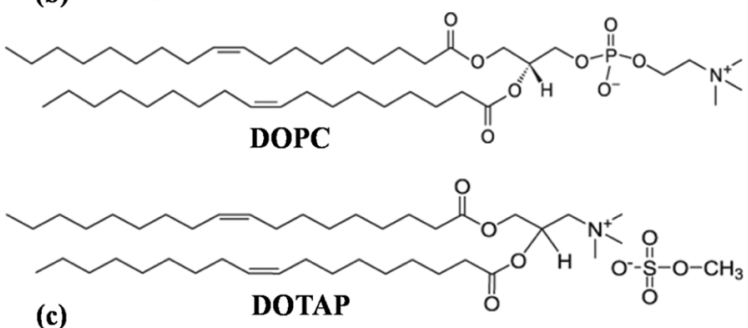

(c)

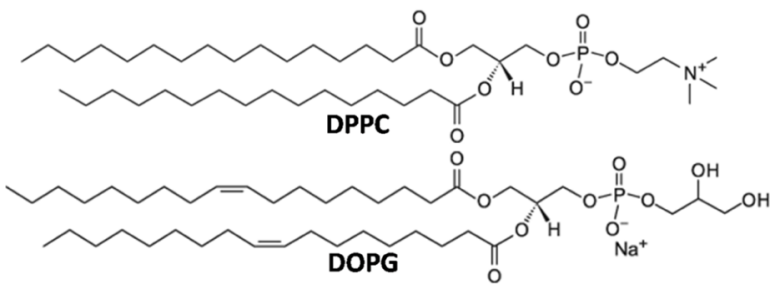

Figure 1. Structure of (a) DS polysaccharide, (b) positively charged mixture of lipids: DOPC and DOTAP (methyl sulfate salt), and (c) negatively charged mixture of lipids: DPPC and DOPG.

Here, we tackle the latter option, analyzing the behavior of membranes decorated with DS. We studied two different kinds of vesicle composition with the aim of shedding light on the DS-lipid interaction and the consequent membrane modulation. The structures of the used lipids are shown in Figure 1.

On one hand, we used membranes composed of 1,2dioleoyl-sn-glycero-3-phosphocholine (DOPC) and 1,2-dioleoyl-3-trimethylammonium-propane (DOTAP) in a 9:1 proportion. PC is the major lipid in mammal cells, and DOPC forms fluid membranes at room temperature due to the unsaturations. Therefore, this kind of lipid is widely used as model for membranes ${ }^{12}$ and for drug delivery. ${ }^{13}$ The addition of a low amount of DOTAP is used in order to anchor anionic polymers and thus confer stability to the vesicle for drug delivery. ${ }^{14}$ This molecule was also used to anchor DNA and other macromolecules. ${ }^{14}$

We also studied DS binding to vesicles composed of 1,2dipalmitoyl-sn-glycero-3-phosphocholine (DPPC) and 1,2dioleoyl-sn-glycero-3-phosphoglycerol (DOPG). These vesicles were studied in order to investigate DS-anionic lipid interaction in membranes with phase coexistence and in the presence of metallic cations because preferential interaction with a denser phase in the presence of $\mathrm{Ca}^{2+}$ was reported. ${ }^{15}$

This lipid composition was shown to form a stable vesicle in the presence of salt. ${ }^{16}$ Phase coexistence was reported for different PC/PG proportions, ${ }^{16}$ as well as interactions between these lipids and $\mathrm{Na}^{+}$and $\mathrm{Ca}^{2+}$ ions. ${ }^{16,17}$ We chose an intermediate 1:1 proportion in which phase coexistence and ion-membrane interaction are certainly established.

First, we tested the polymer-membrane interaction and found that DS binds to both membranes. In the case of the anionic membranes, the interaction was favored by the presence of high amounts of sodium ions or a moderate concentration of calcium ions. It was reported that $\mathrm{PC} / \mathrm{PG}$ membranes bind to $\mathrm{Ca}^{2+}$ with high affinity and to $\mathrm{Na}^{+}$with lower affinity, ${ }^{18-20}$ while DS interacts with both ions (with a higher affinity for $\left.\mathrm{Ca}^{2+}\right) \cdot{ }^{21,22}$ Therefore, we propose that a bridge-like interaction mediated by both metal ions $\left(\mathrm{Na}^{+}\right.$or $\mathrm{Ca}^{2+}$ ) occurs, as previously observed with $\mathrm{Ca}^{2+}$ in PC membranes, ${ }^{22,23}$ PC/PA membranes ${ }^{24}$ and also in DMPG monolayers. ${ }^{15}$ Our experiments indicate that the DS- $\mathrm{Ca}^{2+}-$ PG bridge corresponds to a dynamic equilibrium. DS partially displace $\mathrm{Ca}^{2+}$ ions bound to the membrane, forming a loosely packed ionic layer. The optimal polymer-membrane interaction occurs for $2-3 \mathrm{SO}_{3}^{-}$per $\mathrm{Ca}^{2+}$ ion. Additionally, we found that the polymer interacts selectively with the denser regions of the anionic membrane, indicating that this phase is a better platform for the polymer coating.

Subsequently, we analyzed the effect of the polymermembrane interaction on the liposome permeability, stability against detergents, and membrane rigidity. The results indicate that the presence of a DS-coating surface do not alter efficiently the membrane mechanical properties, suggesting that the proposed effects in drug targeting may be related rather with a protection of the liposome from the biochemical agents present in the organisms.

\section{RESULTS}

2.1. DS Binds to PC/PG and DOTAP/PC Membranes. We first tested whether DS binds to monolayers of the different lipid mixtures at the air-water interface using Brewster Angle Microscopy (BAM). The reflectivity (Rp) of the air-water interface at the Brewster angle increases when a film with refractive index different from that of water and air is present. $\mathrm{Rp}$ depends on the thickness and the refractive index of the film; ${ }^{25}$ high Rp values correspond to thick films with high refractive indexes. Because the hydrocarbon chains of lipid films in a dense phase are more stretched than in a disordered phase, denser phases are thicker. Additionally, the refractive index of dense phases is higher than for disordered ones, ${ }^{26}$ and thus, $\mathrm{Rp}$ is high for regions of lipids in a closely packed state than for less-packed phases, which translate to brighter gray levels in the BAM images.

Because we do not know the refractive index of the investigated films, we compared the Rp value in the absence and in the presence of the polymer and analyzed the observed changes as a qualitative measurement of the film thickening caused by the polymer-membrane interaction. ${ }^{15}$

Figure 2 shows BAM images of monolayers composed of DOPG/DPPC 1:1 (PG/PC from now on) in the absence and presence of DS, with and without $\mathrm{CaCl}_{2}$. $\mathrm{BAM}$ images indicated the presence of segregated patches of coexisting phases on both, $\mathrm{NaCl}$ and $\mathrm{CaCl}_{2}$ subphases (brighter regions correspond to the condensed phase regions). The $\mathrm{Rp}$ value was determined in both phases, and thus, two values of $\mathrm{Rp}$ are plotted in Figure 3, for each condition. These experiments were performed at $15{ }^{\circ} \mathrm{C}$, because at $23{ }^{\circ} \mathrm{C}$ domains were too small for a suitable determination of $\mathrm{Rp}$ inside the domains. The anionic monolayers showed higher $\mathrm{Rp}$ values on $\mathrm{DS}$ solutions only in the liquid-condensed regions, indicating that the polymer interacts selectively with the lipids in this phase state. Because of this preferential interaction, the surface pressure corresponding to the apparition of the liquidcondensed domains decreased in the presence of DS (see Table S1) as previously reported for DPPC, DPPA, ${ }^{24}$ and DMPC monolayers. ${ }^{27}$

The increase in $\mathrm{Rp}$ when DS was added was higher on $\mathrm{CaCl}_{2}$ than on $\mathrm{NaCl}$. Similar Rp increases were achieved with high 


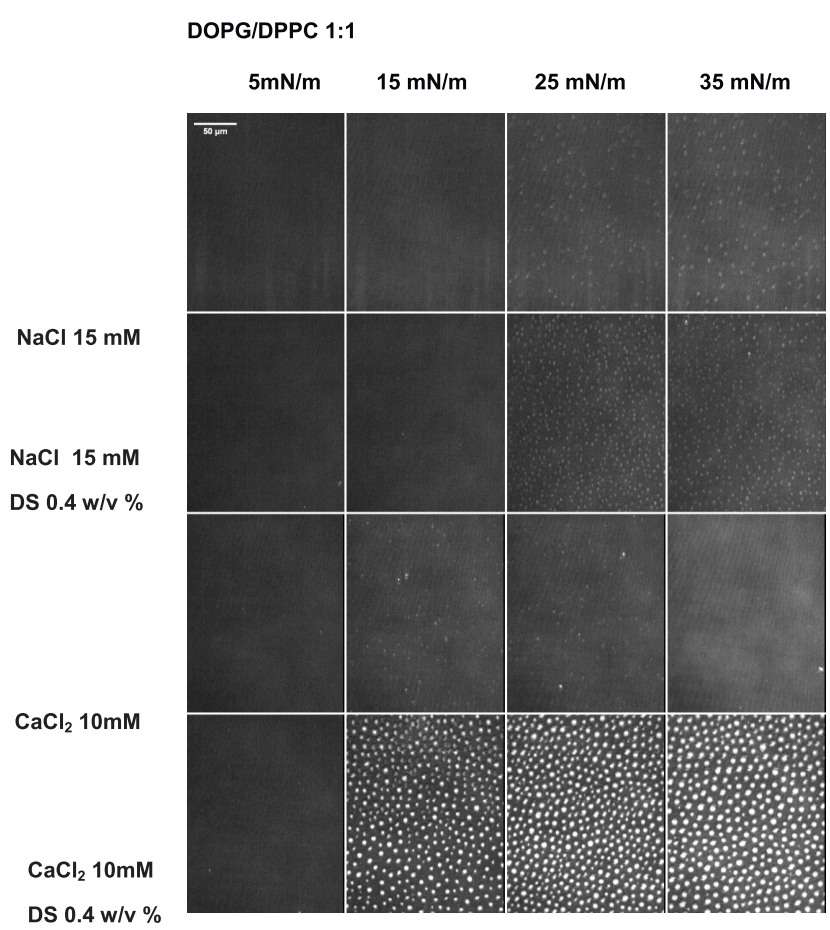

Figure 2. Representative BAM images of monolayers composed of DOPG/DPPC 1:1 in the absence and in the presence of $0.4 \mathrm{w} / \mathrm{v} \%$ of DS at the indicated surface pressures. The gray levels were rescaled from the original $0-255$ range to $9-52$ for better visualization. The brighter regions correspond to regions of lipids in the condensed phase.
$\mathrm{NaCl}$ and low DS concentrations $(145 \mathrm{mM} \mathrm{NaCl}+0.1 \% \mathrm{DS}$, Figure S4) or with low $\mathrm{NaCl}$ and high DS concentrations (10 $\mathrm{mM} \mathrm{NaCl}+0.4 \%$ DS, Figure 2)

The demixing process was not detected in the compression isotherms, and the general behavior of the film upon compression did not change when the polymer was under the lipid monolayer (see Supporting Information S1). This indicates that the polymer binds to the membrane externally, without incorporating between the acyl chains, in the same manner than with DMPG monolayers the larger affinity of DS for the denser phase indicates that this phase is a better platform for the formation of the polymer sublayer.

The domains in a DPC/DOPG mixture are expected to be enriched in the lipid that forms the denser monolayers, which in this case is the PC component. We have previously shown that DS does not influence compression isotherms and fluidity of DPPC monolayers in the absence of $\mathrm{Ca}^{2+}$, while in its presence only a slight decrease in the surface pressure for the phase transition (from 6 to $4 \mathrm{mN} / \mathrm{m}$, see Table S1) was detected without viscosity changes. ${ }^{15}$ Thus, we have concluded that DS binds weakly to DPPC monolayers in the condensed phase state. This was explained considering a lower entropy decrease upon adsorption to these regions of the monolayer because these regions are already ordered before the polymer binding. Similar effects have been reported in the interaction of peripheral proteins with membranes. ${ }^{28}$

All in all, the results shown here are intriguing because they indicate that DS binds selectively to regions enriched in PC and depleted in PG, in spite that pure DPPC experiments showed low changes in the presence of DS. Therefore, we performed BAM experiments for DPPC monolayers over DS
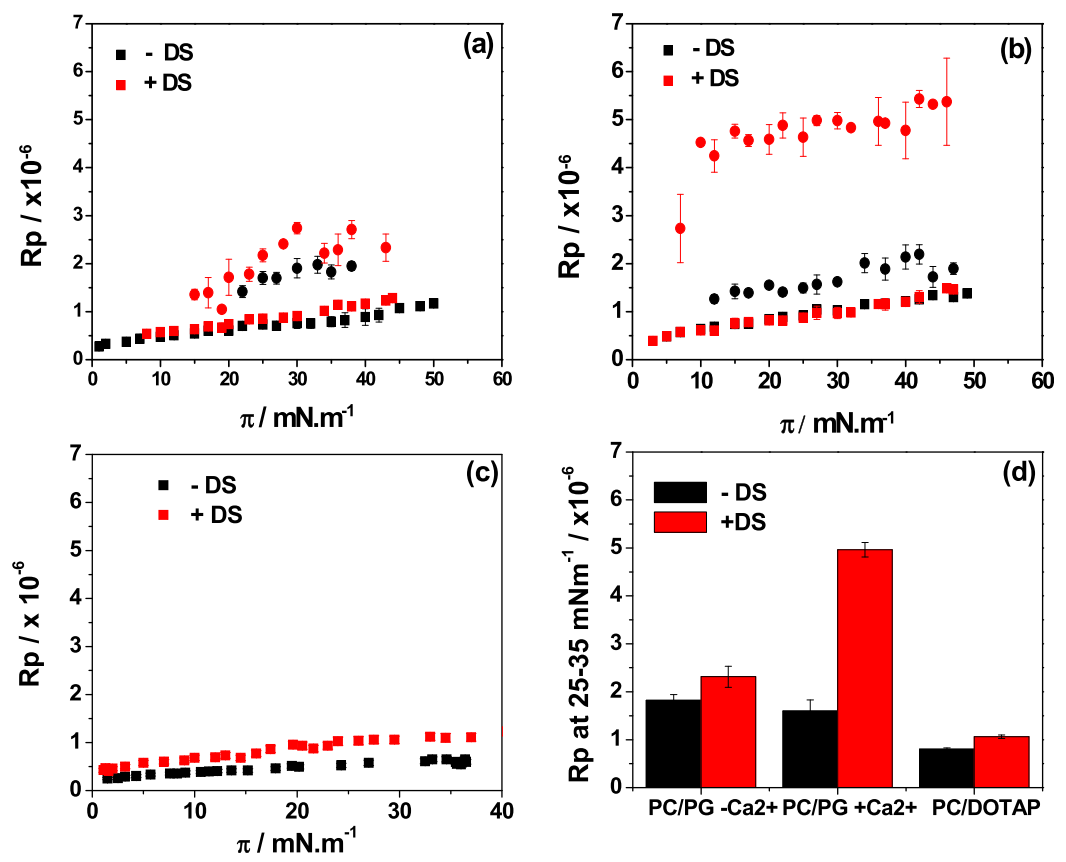

Figure 3. Average reflected light intensity of BAM images as those shown in Figure 2 as a function of surface precision for (a,b) DOPG/DPPC 1:1 in the absence (black) and in the presence of $0.4 \mathrm{w} / \mathrm{v} \%$ DS (red) and (c) DOTAP/DOPC 1:9 monolayer in the absence (black) and in the presence of $0.1 \mathrm{w} / \mathrm{v} \%$ DS (red). Subphase composition: (a) $15 \mathrm{mM} \mathrm{NaCl}$ at $15{ }^{\circ} \mathrm{C}$, (b) $10 \mathrm{mM} \mathrm{CaCl}_{2}$ at $15{ }^{\circ} \mathrm{C}$, and (c) $145 \mathrm{mM} \mathrm{NaCl}$ at $23{ }^{\circ} \mathrm{C}$. The $\mathrm{Rp}$ values correspond to regions of the monolayer in the expanded phase $(\boldsymbol{\square})$ or in the condensed phase $(\bullet)$. Each data correspond to average $\pm \mathrm{SD}$ of six different regions in at least four images. (d) Mean value of Rp in the range of $25-35 \mathrm{mN} / \mathrm{m}$ for each monolayer condition in the absence (black) and in the presence (red) of the corresponding concentration of DS. For the mixture of DOPG/DPPC 1:1, the mean values of Rp were taken from the condensed phase. The mean values in the absence and presence of DS are statistically different in the three conditions analyzed. In all conditions, the p values are lower than 0.0001 for $\alpha=0.05$. 

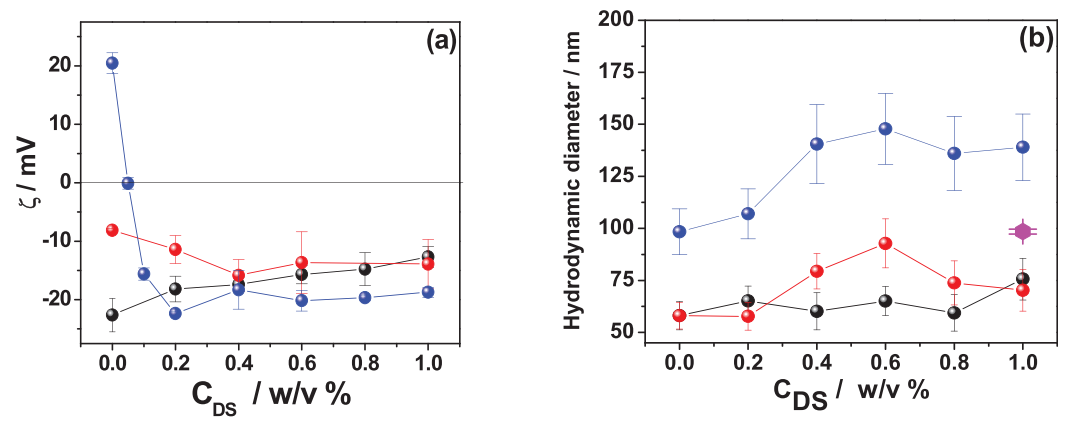

Figure 4. Representative experiments showing the zeta potential values $(\zeta)$ (a) or the average hydrodynamic diameter $\left(d_{\mathrm{H}}\right)(\mathrm{b})$ as a function of the DS concentration. Composition: DOPG/DPPC 1:1 in NaCl $15 \mathrm{mM}$ (black), $\mathrm{CaCl}_{2} 10 \mathrm{mM}$ (red) and $\mathrm{CaCl}_{2} 25 \mathrm{mM}$ (magenta); and DOTAP/ DOPC 1:9 in $\mathrm{NaCl} 15 \mathrm{mM}$ (a-blue) and $145 \mathrm{mM}$ (b-blue). The error bars represent the standard deviation of two independent experiments.

with and without $\mathrm{Ca}^{2+}$. The results are shown in $\mathrm{SM}$, in comparison with similar experiments using PC/PG (Figures S3 and S4). An addition of $0.1 \%$ DS induced an increase in Rp (only in the domains) of similar magnitude than that observed for PC/PG monolayers. It has to be remarked here that these values are not a quantitative measurement of the DSmembrane affinity because the refractive index of the lipid layer and of the DS layer may be different in each system. The refractive index of the polymer layer is expected to depend on the degree of hydration of the polymer at the interface, as well as on its conformation, which may vary from a system to the other, and therefore, we cannot make a quantitative comparison between both systems.

The Rp values of DPPC on DS indicate that PC is able to interact with DS trough an ion metal-bridge even in the absence of PG. We next compared the surface pressure at which domains appear in each system when DS is added (see Table S1) and found that for DPPC, in the absence of $\mathrm{Ca}^{2+}$, this parameter was unaffected, while a $2 \mathrm{mN} / \mathrm{m}$ decrease was detected in the presence of $\mathrm{Ca}^{2+}$. On the contrary, in the PG/ $\mathrm{PC}$ mixture, the demixing surface pressure decreases $5 \mathrm{mN} / \mathrm{m}$ at both conditions (with or without $\mathrm{Ca}$ ). This suggest that the presence of PG inside the domains favors DS binding.

The cationic membranes composed of DOTAP/DOPC 1:9 (DOTAP/PC from now on) were in a liquid-expanded state in the whole compression isotherm (see Figure S1), and the polymer increased the $\mathrm{Rp}$ values (see Supporting Information S2) at all surface pressures (Figure 3c), indicating polymerlipid interactions at all lipid densities, even in a disordered phase state.

Our aim in this work was to study the binding properties of DS on vesicles potentially useful for drug delivery, and thus, we now focus on the $\mathrm{Rp}$ values at conditions comparable to those of bilayers, that is, high surface pressures. ${ }^{29,30}$ Figure $3 \mathrm{~d}$ shows the average $\mathrm{Rp}$ values at $25-35 \mathrm{mN} / \mathrm{m}$ for both membrane composition at each investigated condition (for the anionic membranes, Figure $3 \mathrm{~d}$ only depicts the values for $\mathrm{Rp}$ in the condensed phase). An increment in Rp when DS was present was observed in all cases.

The interaction between DS and anionic membranes ${ }^{15,24}$ and with $\mathrm{PCs}^{22,23}$ was proposed to occur through the formation of a $\mathrm{Ca}^{2+}$-bridge when this bivalent ion is present. In the absence of $\mathrm{Ca}^{2+}$, however, DS-membrane binding was also detected, and therefore, we propose that this interaction occurs by an analogous (though weaker) $\mathrm{Na}^{+}$-bridge favored by DOPG molecules. A weaker interaction is expected as this metal ion binds to PG/PC membranes with a 30 times lower affinity than $\mathrm{Ca}^{2+}$ ions $^{19}$ and with DS, also with a lower affinity than with $\mathrm{Ca}^{2+}$ ions. ${ }^{31}$

The behavior of molecules in bilayers and monolayers is comparable but not equal, ${ }^{32,33}$ and therefore, we subsequently studied the polymer-membrane interaction in large unilamellar vesicles (LUVs), by the determination of zeta potential and hydrodynamic size. Zeta potential measures the electrostatic potential at the slipping plane, which depends on the surface charge density (and thus on the surface charge and on the vesicle size) and also on the double layer potential, which decreases exponentially from the vesicle surface to the bulk of the solution according to the Debye length (that in turn depends on the salt concentration of the dispersing solution). ${ }^{34}$ As higher the vesicle charge density and lower the ionic strength of the dispersion solution, higher the absolute value of the zeta-potential.

The results for vesicles incubated in solutions with increasing DS concentrations are shown in Figures 4 and S6. The cationic vesicles showed positive values of zeta potential and average sizes of $100 \mu \mathrm{m}$ and acquired negative zeta potential values when they were incubated in DS solutions, with a concomitant increment in the vesicle hydrodynamic diameter $\left(d_{\mathrm{H}}\right)$ up to about $150 \mu \mathrm{m}$, indicating DS-membrane binding.

The anionic vesicles depicted negative zeta potential values both in the presence and in the absence of $\mathrm{Ca}^{2+}$, but a decrease in the negative charge (from -23 to $-8 \mathrm{mV}$ ) was observed when $\mathrm{Ca}^{2+}$ was present, indicating a partial charge neutralization of the PG groups as previously reported. ${ }^{18,19}$ The incubation of the anionic LUV's in solutions with increasing concentrations of DS without $\mathrm{Ca}^{2+}$ led to a subtle but constant decrease in the absolute value of the zeta potential (from -23 to $-13 \mathrm{mV}$ ) with no noticeable changes in $\mathrm{d}_{\mathrm{H}}$. This shift toward zero in zeta potential may be due to thinning of the double layer region caused by the increment in ionic strength when the polymer with their counter ions is added, ${ }^{34}$ because polymer binding would make this value more negative. The slipping plane is proposed to be located at distances of $10^{-1}$ $\mathrm{nm}$ from the charged surface of the membranes, ${ }^{35}$ while the Debye length changes from 2 to $10^{-3} \mathrm{~nm}$ for ionic strength changes of $20-50 \mathrm{mM}$ (values in the range that we are evaluating). Therefore, at the investigated conditions, small changes in the surface charge density of the LUVs due to DS addition may be hidden by changes in the Debye length.

The incubation of the anionic LUVs in solutions with $\mathrm{Ca}^{2+}$ and increasing concentrations of DS led to a non-monotonic behavior in $d_{\mathrm{H}}$ and also in zeta potential, although the latter was very subtle. The zeta potential started at $-8 \mathrm{mV}$, decreased 

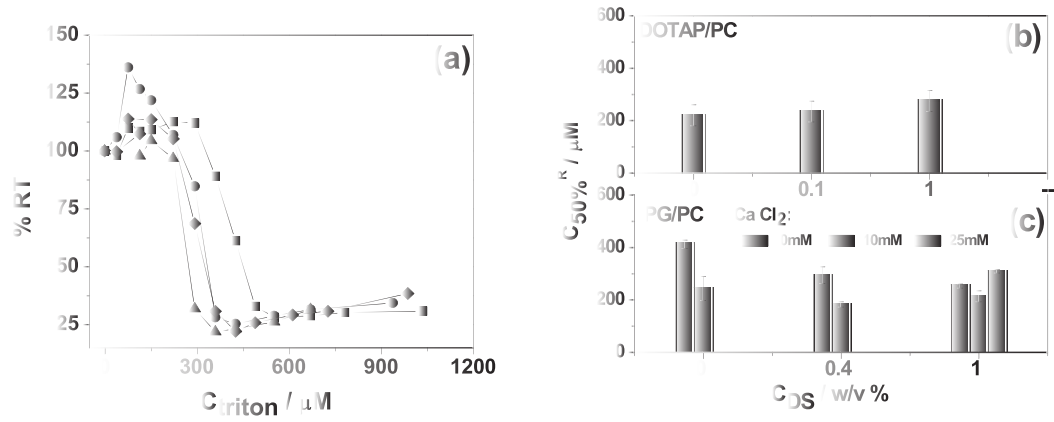

Figure 5. (a) Representative experiments showing the percent of relative turbidity (\% RT) vs Triton X-100 concentration for LUVs composed of DOPG/DPPC 1:1. Composition of the solutions: $\mathrm{CaCl}_{2} 10 \mathrm{mM}+\mathrm{DS} 0 \mathrm{w} / \mathrm{v} \%$ (black), DS $0.4 \mathrm{w} / \mathrm{v} \%$ (red), DS $1 \mathrm{w} / \mathrm{v} \%$ (blue), and CaCl 25 $\mathrm{mM}+\mathrm{DS} 1 \mathrm{w} / \mathrm{v} \%$ (magenta). (b) $C_{50 \%}^{\mathrm{R}}$ vs DS concentration for DOTAP/DOPC 1:9 in NaCl $145 \mathrm{mM}$; (c) $C_{50 \%}^{\mathrm{R}}$ vs DS concentration for DOPG/ DPPC 1:1 in $\mathrm{NaCl} 15 \mathrm{mM}$ (red), $\mathrm{CaCl}_{2} 10 \mathrm{mM}$ (blue), and $\mathrm{CaCl}_{2} 25 \mathrm{mM}$ (magenta). The data correspond to average $\pm \mathrm{SD}$ of two independent experiments.
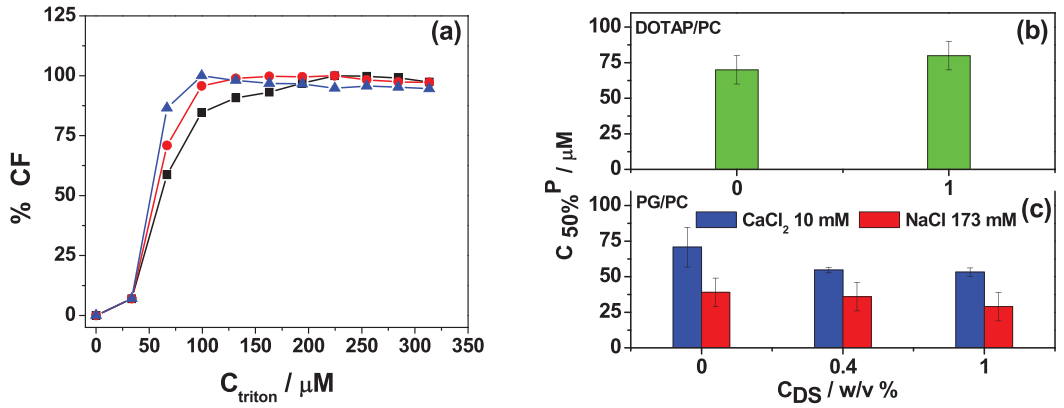

Figure 6. (a) Representative experiments showing the percent of increase in the fluorescence due to the release of carboxyfluorescein (\% CF) vs triton X-100 concentration for LUVs composed of DOPG/DPPC 1:1. Composition of the solutions: $\mathrm{CaCl}_{2} 10 \mathrm{mM}+\mathrm{DS} 0 \mathrm{w} / \mathrm{v} \%$ (black), DS 0.4 $\mathrm{w} / \mathrm{v} \%$ (red), and DS $1 \mathrm{w} / \mathrm{v} \%$ (blue). (b) $C_{50 \%}^{\mathrm{CF}}$ for different DS concentrations for DOTAP/DOPC 1:9 in NaCl $173 \mathrm{mM}$. (c) $C_{50 \%}^{\mathrm{CF}}$ for different DS concentrations for DOPG/DPPC $1: 1 \mathrm{in} \mathrm{NaCl} 173 \mathrm{mM}$ in the absence (red) and presence of $\mathrm{CaCl}_{2} 10 \mathrm{mM}$ (blue). The data correspond to average $\pm \mathrm{SD}$ of two independent experiments.

to $-16 \mathrm{mV}$, and afterward, it increased to $-14 \mathrm{mV}$, while $d_{\mathrm{H}}$ first increased $60 \%$ of its initial value, and afterward, it decreased.

Because DS interacts with $\mathrm{Ca}^{2+}$ ions, ${ }^{21,24}$ the non-monotonic behavior may be related with a competition of DS and the lipids (PG group) for the bivalent ion. With this in mind, $d_{\mathrm{H}}$ was determined for $1 \% \mathrm{DS}$ in the presence of $25 \mathrm{mM}$ of $\mathrm{CaCl}_{2}$, which corresponds to a ratio DS/ $\mathrm{Ca}^{2+}$ equal to that in $0.4 \% \mathrm{DS}$ (high value of $d_{\mathrm{H}}$ and minimum of the zeta potential). Under this condition, a high value of $d_{\mathrm{H}}$ was found (magenta symbol in Figure 4), making evident a reversion of the effect found for DS $1 \% \mathrm{CaCl}_{2} 10 \mathrm{mM}$ and matching our hypothesis. The same experiment was not performed by measuring the zeta potential as this parameter also depends on the aqueous ionic strength. ${ }^{34}$

2.2. Effect of DS on the Stability of Vesicles. Detergents are able to disrupt the lamellar structure of membranes, intercalating between the lipid hydrocarbon chains and promoting micellization. ${ }^{36,37}$ We investigated here whether the presence of DS decorating the vesicles was able to generate a protecting layer, promoting on the membrane a higher stability upon detergent addition.

Figure 5a shows representative experiments for PG/PC vesicles in the presence of $\mathrm{Ca}^{2+}$ (for the other systems, see Supporting Information S7). The addition of small volumes of TritonX-100 generated an increase in the measured diffracted light, which was more noticeable for some systems than for others. This increment was not related with shape changes of the vesicles, as dynamic light scattering (DLS) experiments showed similar distributions than in the absence of detergent (see Supporting Information S8 as an example of the performed controls), thus it might be related with detergentinduced changes in the diffraction index. After this increase, the diffracted light decreased abruptly reaching low values (70-80\% lower than the initial values). This decrease was always related with a rupture of the vesicles, as determined by DLS experiments (see representative experiments in Supporting Information S8).

From duplicate experiments, we determined the concentration of TritonX-100 that promoted the rupture of $50 \%$ of the vesicles, that is, a $50 \%$ decrease in the diffracted light $\left(C_{50 \%}^{\mathrm{R}}\right)$, and plotted these values in Figure $5 \mathrm{~b}, \mathrm{c}$. The $C_{50 \%}^{\mathrm{R}}$ values for the bare cationic vesicles were similar to that of the decorated ones, indicating that the polymer did not prevent the vesicle rupture by the detergent (Figure 5a). This suggests that DS covers the vesicles forming a relaxed layer, leaving holes where the detergent is able to access the lipid bilayer. Similar results were found for the PG/PC vesicles in the absence of $\mathrm{Ca}^{2+}$, while in the presence of the bivalent ion, $C_{50 \%}^{\mathrm{R}}$ decreased when DS was added, Figure 5c. This destabilization can be explained considering that $\mathrm{Ca}^{2+}$ ions stabilize the vesicles, notice that in the absence of polymer, the $10 \mathrm{mM}$ of the bivalent ion promotes a 1.7 times increase in $C_{50 \%}^{\mathrm{R}}$. As already proposed, the polymer competes with the lipids for $\mathrm{Ca}^{2+}$, influencing the $\mathrm{Ca}^{2+}-$ membrane properties and translating to a decreased stability upon TritonX-100 addition.

In order to test this proposition, experiments with $1 \%$ DS and $25 \mathrm{mM} \mathrm{Ca}^{2+}$ (the same $\mathrm{Ca}^{2+} / \mathrm{DS}$ ratio as with $10 \mathrm{mM} \mathrm{Ca}^{2+}$ and $0.4 \% \mathrm{DS}$ ) were performed, and the obtained $C_{50 \%}^{\mathrm{R}}$ value 
(a)
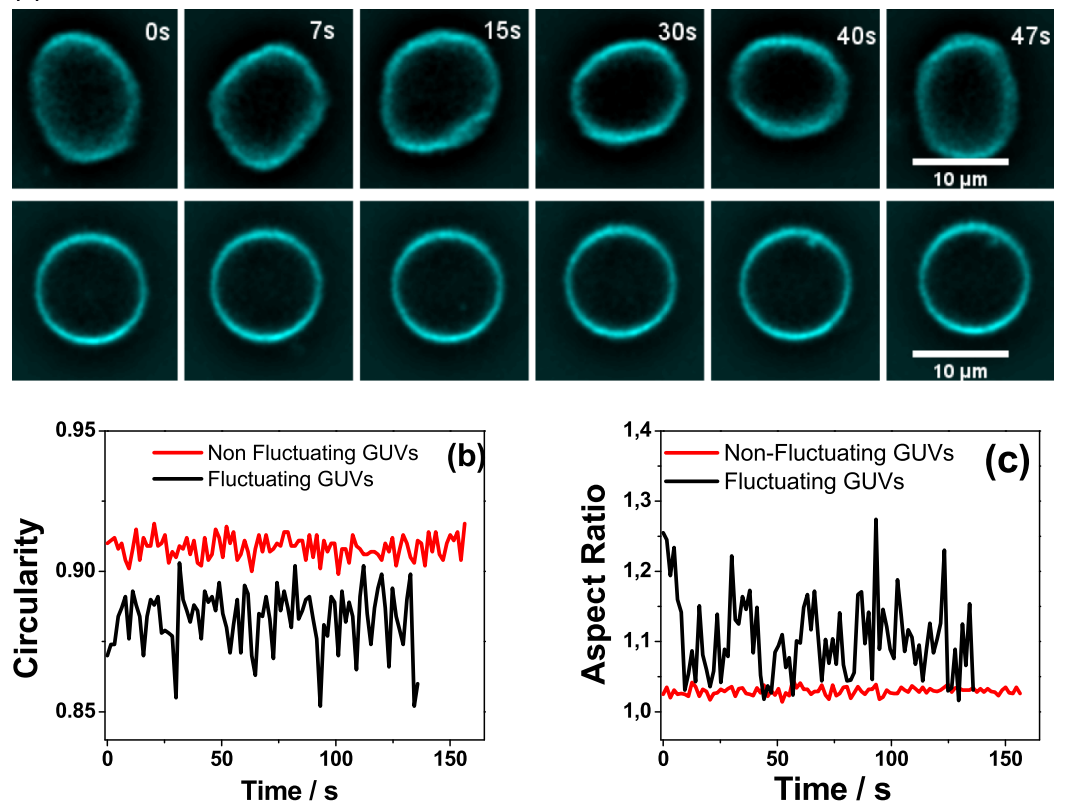

Figure 7. (a) Representative images for fluctuating (upper panelpanel) and nonfluctuating (lower panel) GUV. Representative graphic of circularity (b) and $\mathrm{AR}$ (c) as a function of the time for nonfluctuating (red) and fluctuating (black) GUV.

was similar to that with less polymer but the same $\mathrm{Ca}^{2+} / \mathrm{DS}$ ratio (magenta bar in Figure 5c).

Aside from high vesicle stability upon shocks, low permeability is desired for vesicle-mediated delivery of soluble drugs. Therefore, we tested the effect of DS on the permeability of the vesicles upon addition of sublytic detergent concentrations following the fluorescence of carboxyfluorescein $(\mathrm{CF})$. Figures $6 \mathrm{a}$ and $\mathrm{S} 7$ show representative experiments. The concentrations of TritonX-100 at which 50\% of CF escaped from the vesicles ( $50 \%$ increase in the fluorescence, $C_{50 \%}^{\mathrm{CF}}$ ) are plotted in Figure $6 \mathrm{~b}, \mathrm{c}$. For the cationic vesicles in the absence of polymer and for the anionic vesicles with $\mathrm{Ca}^{2+}$, the $C_{50 \%}^{\mathrm{CF}}$ values were similar, while for the anionic vesicles without $\mathrm{Ca}^{2+}$ it was about half those values, that is, the sensitivity of the permeability upon detergent addition decreased in the presence of $\mathrm{Ca}^{2+}$ for PG/PC membranes. DS did not affect the $C_{50 \%}^{\mathrm{CF}}$ value for the cationic vesicles and the anionic vesicles without $\mathrm{Ca}^{2+}$. When the bivalent ion was present, the addition of DS promoted a slight decrease of $C_{50 \%}^{\mathrm{CF}}$ (from 71 to $53 \mu \mathrm{M}$ ), in concordance with the proposed DS-lipid competition for $\mathrm{Ca}^{2+}$.

\subsection{Shape Fluctuations of Giant Unilamellar Vesicles} (GUVs). In order to study the membrane rigidity in the presence of the polymer, we registered the shape fluctuation of individual GUVs, which depends on the energy cost for this shape change in comparison with the thermal energy.

As the viscosity of the solutions could affect these measurements, we determined the shear viscosities of the different solutions. Similar results were found as detailed in SM5 (Figure S9 and Table S2). Thus, the differences found in the thermal shape fluctuations of the GUVs are not related with the viscosity of the surrounding media but to membrane rigidity.

In order to quantify the effect of the polymer in the vesicle shape and shape fluctuations, we recorded individual GUVs in each condition (100 frames, 38 frames/min). Figure 7 a shows two representative images of GUVs, and Movies S1 and S2 shows the corresponding movies (Supporting Information). The shape fluctuations were analyzed using the morphological package of ImageJ, two shape descriptors were determined: the circularity $(C=4 \pi \cdot$ area/(perimeter $) 2)$ and the aspect ratio ( $A R=$ longer axis length/minor axis length). The values of $C$ and $\mathrm{AR}$ were determined in each frame along the movies, as shown in Figure $7 b, c$. Each vesicle was characterized with a time-average value of circularity (C) and of $\mathrm{AR}$ and its respective standard deviation $\left(\mathrm{SD}_{\mathrm{C}}\right.$ and $\mathrm{SD}_{\mathrm{AR}}$, see Table $\mathrm{S} 3$ ).

The following three different conditions were studied, GUVs composed of: (1) DOTAP/PC in NaCl $145 \mathrm{mM}$, (2) PG/PC in $\mathrm{NaCl} 15 \mathrm{mM}$, and (3) PG/PC in $\mathrm{CaCl}_{2} 10 \mathrm{mM}$. Condition (1) was subjected to two treatments: 0 and $1 \mathrm{w} / \mathrm{v} \%$ of DS, while conditions (2) and (3) to three treatments: $0,0.4$, and 1 $\mathrm{w} / \mathrm{v} \%$ of DS.

For a given condition, we define the treatment that led to the less fluctuating GUV population as the treatment in which the data for $\mathrm{SD}_{\mathrm{C}}$ and $\mathrm{SD}_{\mathrm{AR}}$ yielded the lower value for percentile 95th (P95th, i.e., the value below which $95 \%$ of the data are found $\left.{ }^{38}\right)$. P95th values were determined with a free software (InfoStat). The value of P95th of the $\mathrm{SD}_{\mathrm{C}}$ or $\mathrm{SD}_{\mathrm{AR}}$ data of the less fluctuating treatment was taken as an upper limit (UL), and GUV population was separated in two subgroups: "fluctuating" and "nonfluctuating" vesicles. GUVs with standard deviations higher than the UL value were considered "fluctuating GUVs" and the rest, "nonfluctuating GUVs". Figure 7, Tables S3, and S4 show as example the analysis for GUVs composed of $\mathrm{PG} / \mathrm{PC}$ in $\mathrm{CaCl}_{2} 10 \mathrm{mM}$. The UL value is represented as a magenta line in Figure S10, which in this case corresponds to the P95th value for DS $0.4 \mathrm{w} / \mathrm{v} \%$ (for $\mathrm{SD}_{\mathrm{C}}$ ).

In each condition and treatment, at least 20 GUVs were studied, and the percent of fluctuating GUVs was calculated as

$$
\begin{aligned}
& \text { Percent of fluctuating GUVs } \\
& =\frac{\text { number of fluctuating GUVs }}{\text { number of total GUVs }} \times 100
\end{aligned}
$$


This percent was calculated for two independent experiments and the results shown are the average \pm SD of these two data.

Figure $8 \mathrm{a}-\mathrm{c}$ shows the percent of fluctuating GUVs for different DS concentrations for the different conditions.
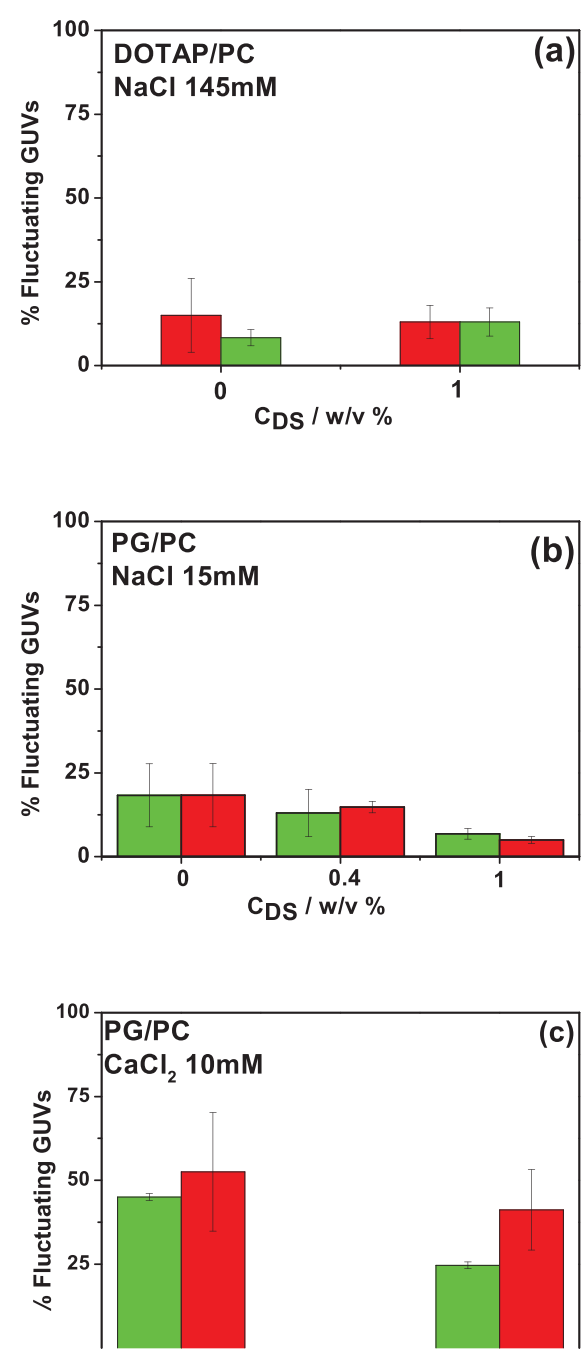

Figure 8. Percent of fluctuating GUVs for different DS concentrations (w/v \%). GUVs are composed of DOTAP/DOPC 1:9 in NaCl 145 $\mathrm{mM}$ (a) or DOPG/DPPC 1:1 in $\mathrm{NaCl} 15 \mathrm{mM}$ (b) or $\mathrm{CaCl}_{2} 10 \mathrm{mM}$ (c). The percent was determined through fluctuations in the circularity (green) or the AR (red). The data correspond to average $\pm \mathrm{SD}$ of two independent experiments.

In the absence of polymer, both membrane compositions showed a low level of fluctuation when dispersed in $\mathrm{NaCl}$ solutions because of the suppression of the undulations that in known to occur in charged membranes caused by the additional energy cost of undulating the Gouy-Chapman double layer. ${ }^{39,40}$ The addition of $\mathrm{Ca}^{2+}$ increased 2.5 times the population of fluctuating anionic vesicles because of the partial neutralization of the membrane charge by this ion. ${ }^{18,19}$

The addition of DS to the dispersing solution did not modify the percent of fluctuating vesicles for GUVs composed of DOTAP/PC (Figure 8a). In this system, the adsorption of DS on the membrane surface modifies the zeta potential values from $\sim+20$ to $\sim-20 \mathrm{mV}$ (i.e., without changes in the absolute value), while $d_{\mathrm{H}}$ increases, and thus probably the charge density at the slipping plane decreases. These changes appear not to affect the thermal fluctuations or, alternatively, the possible changes are counterbalanced by other changes promoted by the DS coating. In this regard, the addition of DS to the cationic liposome solution modifies the membrane thickness (as detected by BAM) and very probably the double layer thickness because of changes in the interfacial electrostatic properties. Both changes have been reported to influence the bending rigidity; a decrease in the double layer thickness or in the surface charge decreases the bending rigidity, ${ }^{39-46}$ while a membrane thickening increases this parameter. ${ }^{45}$

For PG/PC GUVs in the absence of $\mathrm{Ca}^{2+}$, Figure $8 \mathrm{~b}$, the polymer induces a decrease in the population of fluctuating GUVs, suggesting an increase in the membrane rigidity. This may be due to the increment in the effective membrane thickness, as observed by BAM, and/or to a charge increase at the surface as the polymer binds. In this regard, the slight shift toward zero depicted in the zeta potential not necessarily corresponds to a decrease in the surface charge, but it may be due to a decrease in the thickness of the Debye length when the polymer binds to the vesicles, as already pointed out.

In the case of the anionic membranes in the presence of $\mathrm{Ca}^{2+}$, Figure $8 \mathrm{c}, 0.4 \%$ of DS decreased 6 times the population of fluctuating GUVs. This is very probably due to an increase in the surface charge of the membrane by the anionic polymer binding, and an increase in the effective membrane thickness is due to the formation of a PG-Ca-DS layered structure. This is in agreement with the increase in monolayer reflectivity, LUV's hydrodynamic diameter, and zeta potential experiments.

The addition of larger amounts of DS to this system promotes an increase in the fluctuating population, approaching the values obtained for $0 \%$ DS. This is remarkable because a competition between DS and PG for the bivalent ion would lead to a more negatively charged membrane, with a lower population of fluctuating GUVs. The recovery of a highly fluctuating population in the presence of $1 \%$ DS shows that at these conditions the system is not simply composed of anionic $\mathrm{PG} / \mathrm{PC}$ vesicles with the $\mathrm{Ca}^{2+}$ ions sequestered by the polymer.

The experiments shown here allowed to qualitatively test the mechanical properties of the different bilayers in the presence of the polymer. In order to get a more quantitative information of the bending rigidity modulus, more detailed analysis of the contour fluctuations of GUVs using phase-contrast video microscopy can be performed. ${ }^{47-49}$ Alternatively, the pipette aspiration technique ${ }^{50}$ and optical tweezers have been used. ${ }^{51}$

\section{DISCUSSION}

Our results indicate that DS binds to the cationic vesicles, forming a polymer layer close to the polar head-group without incorporating into the membrane and providing the vesicle with a negative surface and a large hydrodynamic diameter. The DS coverage forms a loosely packed layer, which appears not to affect membrane stability upon the tested perturbations, nor their rigidity.

In the case of the anionic vesicles, the affinity of the polymer depends on the ionic composition of the milieu. $\mathrm{Na}^{+}$and $\mathrm{Ca}^{2+}$ are known to interact with $\mathrm{PG} / \mathrm{PC}$ membranes with affinities of 0.6 and $18.8 \mathrm{M}^{-1},{ }^{19,20}$ respectively, leaving a partially neutralized surface. In turn, DS interacts with both cations; with $\mathrm{Ca}^{2+}$, an apparent constant of $24.6 \mathrm{M}^{-1}$ have been reported, ${ }^{21,23}$ while the affinity for $\mathrm{Na}^{+}$is lower. ${ }^{21,23}$ Therefore, we propose that DS binding to the lipid surface occurs through a metal ion-bridge, without penetrating the membranes. 


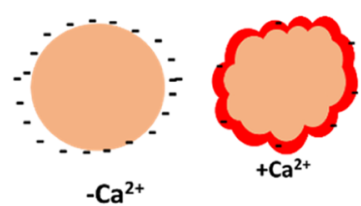

(a)

(b)

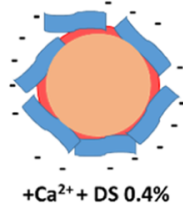

(c)

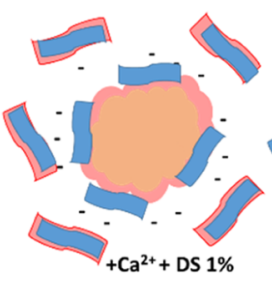

(d)

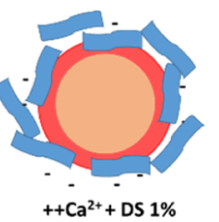

(e)

Figure 9. Scheme of the proposed structures formed by the anionic vesicles and DS: (a) In $\mathrm{NaCl}$ solutions: negatively charged vesicles with small sizes $\left(60 \mathrm{~nm}\right.$ ), rigid and sensitive to detergent addition. (b) In $\mathrm{NaCl}+10 \mathrm{mM} \mathrm{CaCl}_{2}$ solutions: $\mathrm{Ca}^{2+}$ partially neutralizes the membrane charge resulting in fluctuating vesicles with small sizes $(60 \mathrm{~nm})$ and high resistance to detergent addition. (c) In $\mathrm{NaCl}+10 \mathrm{mM} \mathrm{CaCl} 2+0.4 \mathrm{w} / \mathrm{v} \% \mathrm{SD}$ solutions: $\mathrm{Ca}^{2+}$ ions interact with both, DS and the membrane, the negative charge increases and the vesicle + coverage forms a rigid and large ( 80 $\mathrm{nm}$ ) structure, which is sensitive to detergent addition. $\mathrm{Ca}^{2+}$ forms a diffuse layer surrounding the vesicle. (d) $\mathrm{In} \mathrm{NaCl}+10 \mathrm{mM} \mathrm{CaCl}+1 \mathrm{w} / \mathrm{v} \%$ SD solutions: the polymer partially removes $\mathrm{Ca}^{2+}$ ions. Vesicles are of intermediate sizes $(70 \mathrm{~nm})$ and with fluctuation properties between $(\mathrm{b}, \mathrm{c})$. The sensitivity against detergent is also intermediate. (e) In $\mathrm{NaCl}+25 \mathrm{mM} \mathrm{CaCl} \mathrm{C}_{2}+1 \mathrm{w} / \mathrm{v} \% \mathrm{SD}$ solutions: vesicles are large (100 nm), due to a thick layer of polymer and $\mathrm{Ca}^{2+}$ with characteristics similar to that in (c), where the $\mathrm{Ca}^{2+} / \mathrm{DS}$ proportion is the same, and thus, the sensitivity against detergent is similar.

The polymer affected only very slightly or did not affect the tested properties of this vesicles in the absence of $\mathrm{Ca}^{2+}$. On the contrary, DS promoted a destabilizing effect in the presence of the bivalent ion. We propose that this is caused by a competition of the polymer with the lipids for $\mathrm{Ca}^{2+}$, leading to a more loosening $\mathrm{Ca}^{2+} /$ lipid structure, and thus to a less packed lipid structure when DS is present, that resembles that in the absence of $\mathrm{Ca}^{2+}$. Experiments performed with a high $\mathrm{Ca}^{2+} / \mathrm{DS}$ ratio support this hypothesis.

However, the shape fluctuations of the GUVs in the presence of excess of polymer suggest that at these conditions the system is not simply composed of anionic PG/PC vesicles with the $\mathrm{Ca}^{2+}$ ions completely sequestered by the polymer, but that DS partially removes the bivalent ions, leading to a less tight $\mathrm{Ca}^{2+} /$ lipid interaction. Taking together all the results, we propose for the vesicles composed of $\mathrm{PG} / \mathrm{PC}$, the structures schematized in Figure 9.

The optimal $\mathrm{Ca}^{2+} / \mathrm{DS}$ proportion for the $\mathrm{Ca}^{2+} / \mathrm{DS} /$ lipid interaction occurs at $10 \mathrm{mM} \mathrm{Ca}^{2+}$ and $0.4-0.6 \%$ DS. Taking into account the reported range of molecular weights for DS (6500-10000 Da), $0.5 \%$ corresponds to $\sim 0.6 \mathrm{mM}$, that is, to a $\mathrm{Ca}^{2+} / \mathrm{DS}$ molar proportion of $\sim 17$. Because the average percent of sulfur in the polymer is $17 \%$, the optimal interaction occurs for a $\mathrm{Ca}^{2+} / \mathrm{SO}_{3}{ }^{-}$proportion of $1: 2-1: 3$. This indicates that the negative charge of the liposome/DS structure is not completely neutralized by the bivalent ion.

\section{CONCLUSIONS}

The experiments shown in this work aimed at analyzing the reasons for the proposed stabilizing effects of polysaccharides on lipid vesicles potentially useful as vehicles for drug delivery. We found that although DS bound to the tested membranes, this interaction did not promote changes in the stability upon the stresses proved here, nor in the rigidity. Moreover, the stabilization promoted by $\mathrm{Ca}^{2+}$ on $\mathrm{PG} / \mathrm{PC}$ membranes was partially reverted by the addition of DS.

Our results therefore indicate that the main effect of DS on vesicles in drug delivery systems should lay on processes occurring in vivo and not on the physicochemical properties of the preparation. For instance, the polysaccharide covering may promote invisibilization effects to the immune system, which may be due to the similarity between DS and glicasaminoglicans present in the extracellular matrix of the cell.

\section{EXPERIMENTAL SECTION}

5.1. Materials. DOPC; DOPG (sodium salt); DPPC; DOTAP (methyl sulfate salt); and the lipophilic fluorescent probe L- $\alpha$-phosphatidylethanolamine- $N$-(lissaminerhodamine B sulfanyl) (ammonium salt) (egg-transphosphatidylated, chicken) (Rho-PE) were purchased from Avanti Polar Lipids (Alabaster, AL). We studied two lipid mixtures, DOTAP/ DPPC 1:9 and DOPG/DPPC 1:1.

DS sodium salt from Leuconostoc spp $\left(M_{\mathrm{w}} 6500-10000 \mathrm{Da}\right.$, with approximately $17 \%$ of sulfur), $\mathrm{CF}$, polyethylene glycol tert-octylphenyl ether (TritonX-100), and $\beta$-casein from bovine milk were purchased from Sigma-Aldrich (USA).

Lipid solutions were dissolved in chloroform:methanol 2:1. All the solvents and chemicals used were of the highest commercial purity available. The solutions were prepared with deionized water (18 M $\Omega$, with an Osmoion system Apema, Argentina). $\mathrm{NaCl}$ and $\mathrm{CaCl}_{2}$ were purchased from Merck. Indium tin-oxide slides (ITOs) for GUV's formation were from Nanocs (USA).

5.2. Monolayers at the Air-Water Interface. Compression isotherms were performed with a KSV Langmuir balance, using the Wilhelmy method with a platinum plate. The lipid monolayers were formed by spreading the lipids ( 1 $\mathrm{nmol} / \mu \mathrm{L})$ on the desired aqueous solution $(\mathrm{pH} \approx 6$ due to $\mathrm{CO}_{2}$ dissolution) and compressed at $1 \times 10^{-2} \mathrm{~nm}^{2}$ molecule ${ }^{-1}$ $\mathrm{min}^{-1}$. The subphase contained $\mathrm{NaCl} 145 \mathrm{mM}$ in the case of DOTAP/DPPC and $\mathrm{NaCl} 15 \mathrm{mM}$ or $\mathrm{CaCl}_{2} 10 \mathrm{mM}$ for DOPG/DPPC monolayers.

During compression, monolayers were observed using BAM with an EP3 Imaging Ellipsometer (Accurion) equipped with a $20 \times$ objective (Nikon, NA 0.35). The equipment was calibrated with the clean interface before spreading the lipids in order to determine the relation between the average graylevel of the images and the reflected light intensity (Rp). ${ }^{25,52}$ The average gray-levels $( \pm S D)$ were calculated from six different regions corresponding to each phase in at least four images for each condition using ImageJ.

5.3. Giant Unilamellar Vesicles. GUVs were prepared by electroformation. ${ }^{53}$ The lipid solutions $(0.5 \mathrm{mg} / \mathrm{mL})$ doped with $0.5 \mathrm{~mol} \%$ of the fluorescent probe Rho-PE were spread onto two ITO-coated glass slides at $45{ }^{\circ} \mathrm{C}$ and subjected to vacuum for $2 \mathrm{~h}$ for solvent evaporation. Then, lipids were resuspended in a solution of sucrose $300 \mathrm{mM}$ and the desired electrolyte at $60^{\circ} \mathrm{C}$. For vesicles composed of DOTAP/DPPC 
1:9, a $145 \mathrm{mM} \mathrm{NaCl}$ solution was used, while for DOPG/ DPPC 1:1, lower salt content was used $(\mathrm{NaCl} 15 \mathrm{mM})$ because electroformation of GUVs with large percent of charged lipids requires low ionic strength in the aqueous phase. $^{54}$

The electrodes were connected to a homemade wave generator, ${ }^{55}$ and a low-frequency alternate-field was applied for $1 \mathrm{~h}$ at $37^{\circ} \mathrm{C}$. The waveform was modulated with an initial sine wave of $10 \mathrm{~Hz}$ and an amplitude from 0 to $2.6 \mathrm{~V} V_{\mathrm{pp}}$ increasing linearly in $60 \mathrm{~s}$. After that, the amplitude was maintained at 2.6 $\mathrm{V} V_{\mathrm{pp}}$.

The observation chamber was treated with a $10 \mathrm{w} / \mathrm{v} \% \beta$ casein solution, which prevented the GUV rupture on the glass slide. An aliquot of the vesicle's suspension (approximately 25 $\mu \mathrm{L}$ ) was transferred to the observation chamber containing glucose $300 \mathrm{mM}$ and the electrolyte composition of the internal solution. In order to study the effect of the presence of $\mathrm{Ca}^{2+}, 15 \mathrm{mM} \mathrm{NaCl}$ was replaced by $10 \mathrm{mM} \mathrm{CaCl}_{2}$ in some experiments.

GUVs were observed with a fluorescence confocal microscope (Confocal Zeiss LSM5 Pascal) with a $63 \times(1.4$ NA, oil immersion) objective. At least 20 individual GUVs were observed and recorded (100 frames, 38 frames/min).

5.4. Large Unilamellar Vesicles. LUVs were prepared by generating a uniform lipid film on the wall of a glass tube, by solvent evaporation under an $\mathrm{N}_{2}$ flow from a chloroform/ methanol lipid solution. Final traces of solvent were removed incubating the lipids in a high-vacuum chamber during $2 \mathrm{~h}$. Then, the lipids were resuspended in the desired aqueous solution to a final concentration of $0.5 \mathrm{mg} / \mathrm{mL}$. The suspensions were incubated in a $60{ }^{\circ} \mathrm{C}$ water bath for $1 \mathrm{~min}$, vortexed, and submitted to cold incubation for $1 \mathrm{~min}$. This procedure was repeated five times. The resulting multilamellar vesicles were extruded 20 times through a $100 \mathrm{~nm}$ pore filter (Avanti) at room temperature for the cationic mixture and at $60{ }^{\circ} \mathrm{C}$ for the anionic mixture.

LUVs that contained CF were prepared resuspending the lipids in $50 \mathrm{mM} \mathrm{CF}(\mathrm{pH}=8.00)$. At this concentration, the dye is self-quenched. After the extruding process, free CF was removed filtering the LUV's solution through size-exclusion chromatography (Sephadex G-25) with a $173 \mathrm{mM} \mathrm{NaCl}$ solution (isotonic with the LUV interior).

Light dispersion was determined for LUVs prepared in 145 $\mathrm{mM} \mathrm{NaCl}$ (DOTAP/DPPC 1:9) or $15 \mathrm{mM} \mathrm{NaCl}$ (DOPG/ DPPC 1:1), and zeta potential was measured in LUVs prepared in $15 \mathrm{mM} \mathrm{NaCl}$ for all LUV's composition.

Both Nephelometry and fluorescence measurements were acquired with a Cary Eclipse spectrofluorometer (Agilent Technologies) equipped with a thermally controlled multicuvette holder $\left(23^{\circ} \mathrm{C}\right)$, setting the wavelength slit in $5 \mathrm{~nm}$ and using a $2 \mathrm{~mm}$ path cuvette. For Nephelometry, the excitation and emission wavelengths were set at $523 \mathrm{~nm}$, and for fluorescence, the excitation and emission wavelengths were 490 and $515 \mathrm{~nm}$, respectively.

The relative turbidity and fluorescence were measured after each addition of detergent, and the percent of relative turbidity (\% RT), eq 1, and of released CF (\% CF), eq 2, were calculated respectively as

$$
\% \mathrm{RT}=\frac{\mathrm{RT}^{i}}{\mathrm{RT}^{0}} \times 100
$$

$$
\% \mathrm{CF}=\left[\frac{\left(I_{i}-I_{0}\right)}{\left(I_{\max }-I_{0}\right)}\right] \times 100
$$

where the symbol 0 refers to the initial values and $i$ to those after each addition of the detergent. In eq 2, $I_{\max }$ is the maximum fluorescence value reached, corresponding to the highest detergent concentration.

Hydrodynamic size distributions were determined by DLS using a submicron particle sizer (Nicomp 380), and zeta potential determination was performed with a nanoparticle analyzer (HORIBA SZ-100).

The stability of LUVs upon detergent addition was tested through permeability and vesicle rupture, adding increasing volumes of TritonX-100 to the LUV's solutions. Permeability was determined through the fluorescence of the released $\mathrm{CF}$, while vesicle rupture was followed through turbidity measurements using Nephelometry. The diffracted light depends on the size and amount of diffracting particles, and therefore, a decrease in the detected light is observed upon vesicle rupture. ${ }^{56}$

\section{ASSOCIATED CONTENT}

\section{Supporting Information}

The Supporting Information is available free of charge on the ACS Publications website at DOI: 10.1021/acsomega.8b01537.

Fluctuating GUV (real size movie: $20 \mu \mathrm{m} \times 20 \mu \mathrm{m}$, time-length: $136.59 \mathrm{~s}$ ) (AVI)

Nonfluctuating GUV (real size: $20 \mu \mathrm{m} \times 20 \mu \mathrm{m}$, timelength: $157 \mathrm{~s}$ ) (AVI)

Compression isotherms; BAM images and surface pressure for demixing; size distribution of LUVs determined by DLS; rupture and permeability of LUVs upon addition of detergent; determination of the shear viscosity of the used solutions; shear viscosity values; determination of fluctuating GUVs; and average circularity and corresponding standard deviation of circularity (PDF)

\section{AUTHOR INFORMATION}

\section{Corresponding Author}

*E-mail: wilke@mail.fcq.unc.edu.ar. Phone: +54-351-5353855. ORCID

Maria Laura Fanani: 0000-0002-9495-124X

Natalia Wilke: 0000-0002-2342-0193

\section{Notes}

The authors declare no competing financial interest.

\section{ACKNOWLEDGMENTS}

This work was supported by SECyT-UNC, CONICET, and FONCYT (PICT 2012-0344). N.W. and M.L.F. are Career Investigators and C.I.C. is a fellow of CONICET. We thank Dr. Ambroggio for his help with the GUV's experiments, Dr. Gomez for his assistance in the determination of the bulk shear viscosity of the polymer solutions, and Dr. Sampedro and Dr. Mas from the Centro de Microscopia Optica y Confocal de Avanzada de Córdoba (CEMICO), integrated to the Sistema Nacional de Microscopia (SNM-MINCyT), for their help with the microscopy experiments. 


\section{REFERENCES}

(1) Allen, T. M.; Cullis, P. R. Liposomal drug delivery systems: From concept to clinical applications. Adv. Drug Delivery Rev. 2013, $65,36-48$.

(2) Sihorkar, V. V.; Vyas, S. P. Potential of Polysaccharide Anchored Liposomes in Drug Delivery, Targeting and Immunization. J. Pharm. Pharmaceut. Sci. 2001, 4, 138-158.

(3) Alam, M. I.; Beg, S.; Samad, A.; Baboota, S.; Kohli, K.; Ali, J.; Ahuja, A.; Akbar, M. Strategy for effective brain drug delivery. Eur. J. Pharm. Sci. 2010, 40, 385-403.

(4) Mehvar, R. Dextrans for targeted and sustained delivery of therapeutic and imaging agents. J. Controlled Release 2000, 69, 1-25.

(5) Hamidi, M.; Azadi, A.; Rafiei, P. Hydrogel nanoparticles in drug delivery. Adv. Drug Delivery Rev. 2008, 60, 1638-1649.

(6) Tacar, O.; Sriamornsak, P.; Dass, C. R. Doxorubicin: an update on anticancer molecular action, toxicity and novel drug delivery systems. J. Pharm. Pharmacol. 2013, 65, 157-170.

(7) Oppenheim, R. Solid colloidal drug delivery systems: Nanoparticles. Int. J. Pharm. 1981, 8, 217-234.

(8) Ruttala, H. B.; Ramasamy, T.; Gupta, B.; Choi, H.-G.; Yong, C. S.; Kim, J. O. Multiple polysaccharide-drug complex-loaded liposomes: A unique strategy in drug loading and cancer targeting. Carbohydr. Polym. 2017, 173, 57-66.

(9) Heinze, T.; Liebert, T.; Heublein, B.; Hornig, S. Functional Polymers Based on Dextran. In Polysaccharides II; Klemm, D., Ed.; Springer Berlin Heidelberg, 2006; Vol. 205, pp 199-291.

(10) Raveendran, S.; Yoshida, Y.; Maekawa, T.; Kumar, D. S. Pharmaceutically versatile sulfated polysaccharide based bionano platforms. Nanomedicine 2013, 9, 605-626.

(11) Sato, T.; Sunamoto, J. Recent aspects in the use of liposomes in biotechnology and medicine. Prog. Lipid Res. 1992, 31, 345-372.

(12) Rosetti, C. M.; Mangiarotti, A.; Wilke, N. Sizes of lipid domains: What do we know from artificial lipid membranes? What are the possible shared features with membrane rafts in cells? Biochim. Biophys. Acta, Biomembr. 2017, 1859, 789-802.

(13) Drummond, D. C.; Zignani, M.; Leroux, J.-C. Current status of pH-sensitive liposomes in drug delivery. Prog. Lipid Res. 2000, 39, 409-460.

(14) Campbell, R. B.; Balasubramanian, S. V.; Straubinger, R. M. Phospholipid-cationic lipid interactions: influences on membrane and vesicle properties. Biochim. Biophys. Acta, Biomembr. 2001, 1512, 2739.

(15) Cámara, C. I.; Wilke, N. Interaction of dextran derivatives with lipid monolayers and the consequential modulation of the film properties. Chem. Phys. Lipids 2017, 204, 34-42.

(16) Himeno, H.; Shimokawa, N.; Komura, S.; Andelman, D.; Hamada, T.; Takagi, M. Charge-induced phase separation in lipid membranes. Soft Matter 2014, 10, 7959-7967.

(17) Himeno, H.; Ito, H.; Higuchi, Y.; Hamada, T.; Shimokawa, N.; Takagi, M. Coupling between pore formation and phase separation in charged lipid membranes. Phys. Rev. E: Stat., Nonlinear, Soft Matter Phys. 2015, 92, 062713.

(18) Lau, A.; McLaughlin, A.; McLaughlin, S. The adsorption of divalent cations to phosphatidylglycerol bilayer membranes. Biochim. Biophys. Acta, Biomembr. 1981, 645, 279-292.

(19) Macdonald, P. M.; Seelig, J. Calcium binding to mixed phosphatidylglycerol-phosphatidylcholine bilayers as studied by deuterium nuclear magnetic resonance. Biochemistry 1987, 26, $1231-1240$.

(20) Maity, P.; Saha, B.; Kumar, G. S.; Karmakar, S. Binding of monovalent alkali metal ions with negatively charged phospholipid membranes. Biochim. Biophys. Acta, Biomembr. 2016, 1858, 706-714.

(21) Hardikar, V. V.; Matijević, E. Influence of ionic and nonionic dextrans on the formation of calcium hydroxide and calcium carbonate particles. Colloids Surf., A 2001, 186, 23-31.

(22) Huster, D.; Paasche, G.; Dietrich, U.; Zschörnig, O.; Gutberlet, T.; Gawrisch, K.; Arnold, K. Investigation of Phospholipid Area Compression Induced by Calcium-Mediated Dextran Sulfate Interaction. Biophys. J. 1999, 77, 879-887.
(23) Huster, D.; Arnold, K. Ca2+-Mediated Interaction between Dextran Sulfate and Dimyristoyl-sn-Glycero-3-Phosphocholine Surfaces Studied by $2 \mathrm{H}$ Nuclear Magnetic Resonance. Biophys. J. 1998, 75, 909-916.

(24) Santos, H. A.; García-Morales, V.; Roozeman, R.-J.; Manzanares, J. A.; Kontturi, K. Interfacial Interaction between Dextran Sulfate and Lipid Monolayers: An Electrochemical Study†. Langmuir 2005, 21, 5475-5484.

(25) Wilke, N. Chapter Two-Lipid Monolayers at the Air-Water Interface: A Tool for Understanding Electrostatic Interactions and Rheology in Biomembranes. In Advances in Planar Lipid Bilayers and Liposomes; Aleš, I.; Chandrashekhar, V. K., Eds.; Academic Press, 2014; Vol. 20, pp 51-81.

(26) Ducharme, D.; Max, J. J.; Salesse, C.; Leblanc, R. M. Ellipsometric study of the physical states of phosphatidylcholines at the air-water interface. J. Phys. Chem. 1990, 94, 1925-1932.

(27) Zschörnig, O.; Richter, W.; Paasche, G.; Arnold, K. Cationmediated interaction of dextran sulfate with phospholipid vesicles: binding, vesicle surface polarity, leakage and fusion. Colloid Polym. Sci. 2000, 278, 637-646.

(28) Sengupta, P.; Hammond, A.; Holowka, D.; Baird, B. Structural determinants for partitioning of lipids and proteins between coexisting fluid phases in giant plasma membrane vesicles. Biochim. Biophys. Acta, Biomembr. 2008, 1778, 20-32.

(29) Marsh, D. Lateral pressure in membranes. Biochim. Biophys. Acta, Biomembr. 1996, 1286, 183-223.

(30) Demel, R. A.; van Kessel, W. S. M. G.; Zwaal, R. F. A.; Roelofsen, B.; van Deenen, L. L. M. Relation between various phospholipase actions on human red cell membranes and the interfacial phospholipid pressure in monolayers. Biochim. Biophys. Acta, Biomembr. 1975, 406, 97-107.

(31) Hugerth, A.; Sundelöf, L.-O. Effect of Polyelectrolyte Counterion Specificity on Dextran Sulfate-Amphiphile Interaction in Water and Aqueous/Organic Solvent Mixtures. Langmuir 2000, 16, $4940-4945$

(32) Mangiarotti, A.; Caruso, B.; Wilke, N. Phase coexistence in films composed of DLPC and DPPC: A comparison between different model membrane systems. Biochim. Biophys. Acta, Biomembr. 2014, $1838,1823-1831$.

(33) Mangiarotti, A.; Wilke, N. Energetics of the Phase Transition in Free-Standing versus Supported Lipid Membranes. J. Phys. Chem. B 2015, 119, 8718-8724.

(34) Evans, D. F.; Wennerstrom, H. The Colloidal Domain: Where Physics, Chemistry, Biology and Technology Meet, 2nd ed.; Wiley-VCH: New York, 1999.

(35) Eisenberg, M.; Gresalfi, T.; Riccio, T.; McLaughlin, S. Adsorption of monovalent cations to bilayer membranes containing negative phospholipids. Biochemistry 1979, 18, 5213-5223.

(36) Lichtenberg, D.; Ahyayauch, H.; Alonso, A.; Goñi, F. M. Detergent solubilization of lipid bilayers: a balance of driving forces. Trends Biochem. Sci. 2013, 38, 85-93.

(37) Mattei, B.; França, A. D. C.; Riske, K. A. Solubilization of Binary Lipid Mixtures by the Detergent Triton X-100: The Role of Cholesterol. Langmuir 2015, 31, 378-386.

(38) Alejandro, D. R. J., Fernando, C., Alicia, G. L., Margot, T. E., del Pilar, D. M., Walter, R. R., Graciela, B. M. Estadistica para las Ciencias Agropecuarias, 5th ed.; Brujas: Córdoba, 2003; p 16.

(39) Winterhalter, M.; Helfrich, W. Bending elasticity of electrically charged bilayers: coupled monolayers, neutral surfaces, and balancing stresses. J. Phys. Chem. 1992, 96, 327-330.

(40) Vitkova, V.; Genova, J.; Finogenova, O.; Ermakov, Y.; Mitov, M.; Bivas, I. Surface charge effect on the lipid bilayer elasticity. $C R$ Acad. Bulg. Sci 2004, 57, 25-30.

(41) Mitchell, D. J.; Ninham, B. W. Curvature elasticity of charged membranes. Langmuir 1989, 5, 1121-1123.

(42) Pincus, P.; Joanny, J.-F.; Andelman, D. Electrostatic Interactions, Curvature Elasticity, and Steric Repulsion in Multimembrane Systems. Europhys. Lett. 1990, 11, 763-768. 
(43) Song, J.; Waugh, R. E. Bilayer Membrane Bending Stiffness by Tether Formation From Mixed PC-PS Lipid Vesicles. J. Biomech. Eng. 1990, 112, 235-240.

(44) Rowat, A. C.; Hansen, P. L.; Ipsen, J. H. Experimental evidence of the electrostatic contribution to membrane bending rigidity. Europhys. Lett. 2004, 67, 144-149.

(45) Seth, M.; Ramachandran, A.; Leal, L. G. Direct Measurements of Effect of Counterion Concentration on Mechanical Properties of Cationic Vesicles. Langmuir 2013, 29, 14057-14065.

(46) May, S. Curvature elasticity and thermodynamic stability of electrically charged membranes. J. Chem. Phys. 1996, 105, 83148323.

(47) Bouvrais, H.; Holmstrup, M.; Westh, P.; Ipsen, J. H. Analysis of the shape fluctuations of reconstituted membranes using GUVs made from lipid extracts of invertebrates. Biol. Open 2013, 2, 373-378.

(48) Méléard, P.; Gerbeaud, C.; Pott, T.; Fernandez-Puente, L.; Bivas, I.; Mitov, M. D.; Dufourcq, J.; Bothorel, P. Bending elasticities of model membranes: influences of temperature and sterol content. Biophys. J. 1997, 72, 2616-2629.

(49) Engelhardt, H.; Duwe, H. P.; Sackmann, E. Bilayer bending elasticity measured by Fourier analysis of thermally excited surface undulations of flaccid vesicles. J. Phys., Lett. 1985, 46, 395-400.

(50) Fa, N.; Lins, L.; Courtoy, P. J.; Dufrêne, Y.; Van Der Smissen, P.; Brasseur, R.; Tyteca, D.; Mingeot-Leclercq, M.-P. Decrease of elastic moduli of DOPC bilayers induced by a macrolide antibiotic, azithromycin. Biochim. Biophys. Acta, Biomembr. 2007, 1768, 18301838.

(51) Roux, A.; Cuvelier, D.; Nassoy, P.; Prost, J.; Bassereau, P.; Goud, B. Role of curvature and phase transition in lipid sorting and fission of membrane tubules. EMBO J. 2005, 24, 1537-1545.

(52) Mercado, F. V.; Maggio, B.; Wilke, N. Phase diagram of mixed monolayers of stearic acid and dimyristoylphosphatidylcholine. Effect of the acid ionization. Chem. Phys. Lipids 2011, 164, 386-392.

(53) Angelova, M. I.; Soléau, S.; Méléard, P.; Faucon, F.; Bothorel, $\mathrm{P}$. Preparation of giant vesicles by external AC electric fields. Kinetics and applications. In Trends in Colloid and Interface Science VI; Helm, C.; Lösche, M.; Möhwald, H., Eds.; Steinkopff: Darmstadt, 1992; pp 127-131.

(54) Morales-Penningston, N. F.; Wu, J.; Farkas, E. R.; Goh, S. L.; Konyakhina, T. M.; Zheng, J. Y.; Webb, W. W.; Feigenson, G. W. GUV preparation and imaging: Minimizing artifacts. Biochim. Biophys. Acta, Biomembr. 2010, 1798, 1324-1332.

(55) Bellon, J. A.; Pino, M. J.; Wilke, N. Low-cost equipment for electroformation of Giant Unilamellar Vesicles. HardwareX 2018, 4, e00037.

(56) Brasitus, T. A.; Dudeja, P. K.; Worman, H. J.; Foster, E. S. The lipid fluidity of rat colonic brush-border membrane vesicles modulates $\mathrm{Na}+-\mathrm{H}+$ exchange and osmotic water permeability. Biochim. Biophys. Acta, Biomembr. 1986, 855, 16-24. 